\title{
Adverse events associated with immune checkpoint inhibitor treatment for cancer
}

\author{
Khashayar Esfahani MD, Nicholas Meti MD, Wilson H. Miller Jr. MD PhD, Marie Hudson MD MPH
}

Cite as: CMAJ 2019 January 14;191:E40-6. doi: 10.1503/cmaj.180870

I nhibitors against cytotoxic T-lymphocyte-associated protein 4 and programmed cell death 1 immune checkpoints are novel agents that modulate immune pathways and enhance anti-tumour immunity. Their use has set new standards in the treatment of many cancer types. Some patients with tumours previously deemed incurable are now able to achieve long-term remissions. ${ }^{1,2}$ Immune checkpoint inhibitors are increasingly used, either as stand-alone treatments or in combination with chemotherapy, surgery and radiotherapy, not only for patients with refractory metastatic cancer, but also earlier in the cancer cycle as adjuvant and neoadjuvant treatments. Although these treatments are usually well tolerated, severe and, rarely, fatal adverse events have occurred, especially if not promptly recognized and treated. These reactions, called immune-related adverse events, are the consequence of off-target immune attack on the hosts' healthy tissues. Given the wide spectrum of sometimes unconventional clinical presentations and response to standard treatments, physicians must maintain a high level of clinical suspicion of immune-related adverse events when managing patients who are treated with immune checkpoint inhibitors. The objective of this review is to provide the general clinician with the necessary tools to recognize, understand and begin management of immune-related adverse events. Our search for the evidence supporting this review is detailed in Box 1 .

\section{What are immune checkpoint inhibitors and how do they work?}

The idea of exploiting the host immune system to treat cancer relies on the insight that the immune system can eliminate malignant cells in a process termed immune surveillance. ${ }^{3}$ During this

\section{Box 1: Evidence used}

We searched PubMed from 2010 to 2018 for articles about immunerelated adverse events and immune checkpoint inhibitors and selected randomized controlled trials, systematic reviews, observational studies and case reports. We also reviewed the bibliographies of high-impact articles, such as those of the American Society of Clinical Oncology and European Society for Medical Oncology guidelines for the management of immune-related adverse events.

\section{Key points}

- Immune-related adverse events can occur in any organ at any time during treatment, and possibly after discontinuation of immune checkpoint inhibitors.

- The most important step in managing immune-related adverse events is to recognize them promptly and administer corticosteroids for grade 2 or higher reactions.

- Management of immune-related adverse events is complex, requiring the input of a multidisciplinary team.

- Substantial research is yet to be done on the predictive biomarkers of immune-related adverse events and on their optimal management.

process, positive and negative immune checkpoints ensure a balance between the hosts' defence against tumour antigens and autoimmunity. Tumour cells can distort some of these signals, allowing the cells to escape immune destruction and progress to cancer. ${ }^{4}$ Cytotoxic T-lymphocyte-associated protein 4 and programmed cell death 1 inhibitors are 2 important negative immunomodulatory checkpoints; they are T-cell surface receptors that, when engaged, turn off immune function at different stages of the immunity cycle (Figure 1). Their inhibition thereby allows for ongoing T-cell activation and enhances anti-tumour immunity.

Health Canada's clinical indications for the use of immune checkpoint inhibitors are summarized in Table 1. Because more than a thousand clinical trials are underway assessing different immune checkpoint inhibitors alone or in combination with other standard cancer therapies such as chemotherapy, surgery and radiotherapy, the number of approved indications and patients exposed to these treatments is expected to rise substantially in coming years.

\section{Why do immune-related adverse events occur?}

If immune tolerance is defined as a state of unresponsiveness of the immune system toward antigens or tissues able to elicit an immune response, immune-related adverse events represent the other end of the spectrum, where an activated immune system 

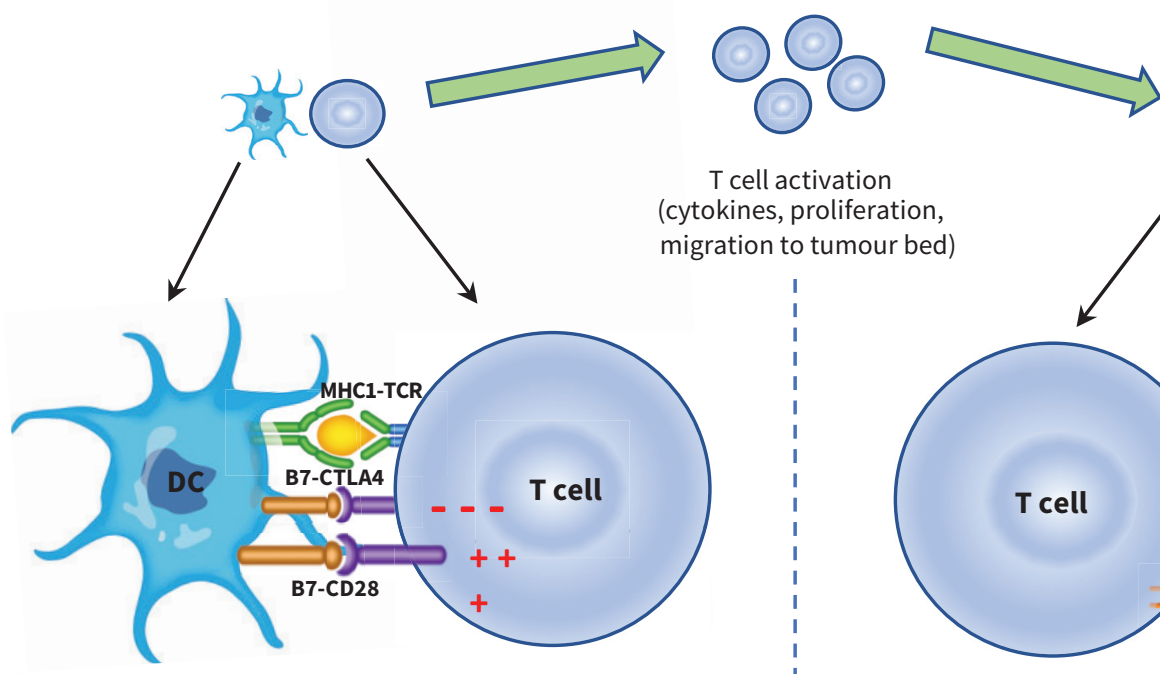

T cell activation (cytokines, proliferation, migration to tumour bed)

I
I
I
I
I
I
I
I
I
I

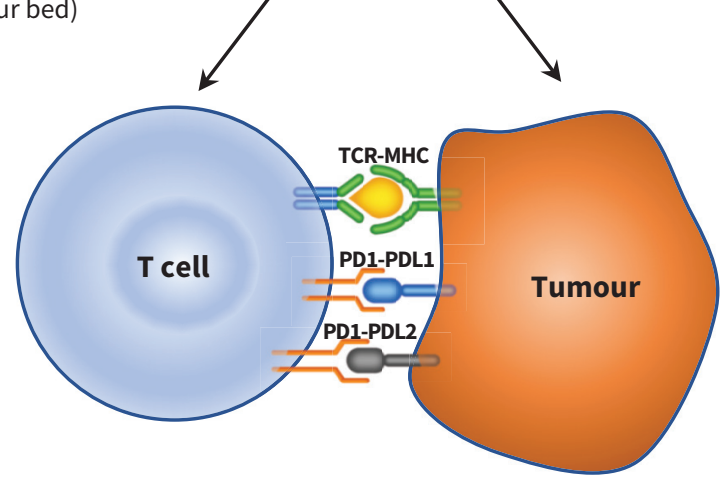

Figure 1: A) Antigen presenting cells (APC), such as dendritic cells (DC), scout the human body, sampling different antigens, including tumour-associated antigens, which are then processed and presented through the major histocompatibility (MHC) complex. In a priming phase that takes place in peripheral lymph nodes, APC educate and activate antigen-specific T cells. T cell activation is kept in check by cytotoxic T-lymphocyte-associated protein 4 (CTLA4), a negative regulator of T-cell function. This negative signal can be blocked by CTLA-4 inhibitors (i.e., ipilimumab), allowing ongoing T-cell activation and migration to the tumour bed. B) Activated T cells also express programmed cell death 1 (PD1), and its ligands, PDL1 and PDL2, are commonly expressed on APC. PD1 and PDL1/PDL2 interaction also inhibits T-cell responses. Tumours can express PDL1 and PDL2 receptors, and engagement of these receptors with the PD1 receptor on T cells turns off T cells and allows tumours to escape destruction. These immune checkpoints can be blocked by PD-1 inhibitors (i.e., nivolumab, pembrolizumab) or PDL-1 inhibitors (i.e., atezolizumab, avelumab and durvalumab). CTLA-4 inhibition results in more widespread and severe immune-related adverse events (irAE), as it regulates T-cell function early in the immunity cycle. Blocking PD-1, on the other hand, is more specific to the tumour microenvironment and, in general, results in less widespread and severe irAE. Note: $C D 28=$ cluster of differentiation $28, \mathrm{TCR}=\mathrm{T}$-cell receptor.

reacts against both tumour antigens and antigens on healthy tissues. This process is tightly regulated by a complex network of cell surface receptors and signalling pathways. By blocking the inhibitory signals, immune checkpoint inhibitors can sway immune responses away from tolerance and in favour of an activated state. ${ }^{5}$ Although this is useful for targeting cancer, an immune episode targeted to healthy tissue is also possible. As such, both cytotoxic T-lymphocyte-associated protein 4 and programmed cell death 1 inhibitors have been associated with inflammatory or autoimmune reactions of the human body's organs (Figure 2). The rates of various immune-related adverse events may differ depending on the particular drug, but the full spectrum of these adverse events can occur with all drugs (Table 2). ${ }^{7}$

Similarly, although some temporal patterns have emerged for each specific immune-related adverse event, it is important to highlight that any such adverse event can occur at any time during treatment. Some cases of autoimmune disease have been attributed to immune checkpoint inhibitors months after cessation of treatment. ${ }^{8}$ Clinicians must be on the lookout for any new symptoms reported by patients who have been exposed to immune checkpoint inhibitors. Early recognition is key, as immune-related adverse events are usually reversible, even when severe in presentation.

\section{How are immune-related adverse events diagnosed and managed?}

We previously reported the case of a patient who developed cerebritis, which rapidly evolved to a profound comatose state after delays in consultation with the treating oncology team and administration of corticosteroids. ${ }^{9}$ This example highlights a critical step in the management of immune-related adverse events, which is early recognition and prompt administration of treatment. Patients may present to their primary care physician or to the emergency department outside their routine oncological visits. As such, frontline health care workers may be involved in the initial assessment and management of immune-related adverse events and are encouraged to rapidly consult with the treating oncologists.

Both the American Society of Clinical Oncology and the European Society of Medical Oncology have released comprehensive clinical guidelines on the diagnosis and management of immune-related adverse events..$^{10,11}$ In summary, adverse events are graded according to the Common Terminology Criteria for Adverse Events (Table 3). ${ }^{12}$

Principles of treatment are outlined in Figure 3, which is adapted from the latest American Society of Clinical Oncology guideline. ${ }^{10}$ Close observation and symptomatic management without interruption of immune checkpoint inhibitors are recommended for grade 1 reactions. 
Table 1: Health Canada-approved indications for immune checkpoint inhibitors in Canada (as of December 2018)*

\begin{tabular}{|c|c|c|c|c|c|c|c|c|}
\hline Agent & $\begin{array}{l}\text { Advanced } \\
\text { melanoma }\end{array}$ & $\begin{array}{l}\text { Advanced } \\
\text { NSCLC }\end{array}$ & $\begin{array}{l}\text { Advanced } \\
\text { RCC }\end{array}$ & $\begin{array}{l}\text { Advanced } \\
\text { SCCHN }\end{array}$ & $\begin{array}{l}\text { Advanced } \\
\text { bladder } \\
\text { cancer }\end{array}$ & $\begin{array}{l}\text { Merkel cell } \\
\text { cancer }\end{array}$ & $\begin{array}{l}\text { Hepato- } \\
\text { cellular } \\
\text { carcinoma }\end{array}$ & $\begin{array}{l}\text { Hodgkin } \\
\text { lymphoma }\end{array}$ \\
\hline \multicolumn{9}{|l|}{ CTLA-4 inhibitor } \\
\hline Ipilimumab & $\begin{array}{l}\text { All lines of } \\
\text { treatment }\end{array}$ & & & & & & & \\
\hline \multicolumn{9}{|l|}{ PD-1 inhibitors } \\
\hline Pembrolizumab & $\begin{array}{l}\text { 1st-line } \\
\text { 2nd-line }\end{array}$ & $\begin{array}{l}\text { 1st-line: }(\geq 50 \% \\
\text { PDL-1+) } \\
\text { 2nd-line: } \\
\text { ( } \geq 1 \% \text { PDL-1+) }\end{array}$ & & & 2nd-line & & & $\begin{array}{l}\text { Post- } \\
\text { autologous } \\
\text { stem cell } \\
\text { transplant }\end{array}$ \\
\hline Nivolumab & $\begin{array}{l}\text { 1st-line } \\
\text { 2nd-line }\end{array}$ & 2nd-line & 2nd-line & 2nd-line & & & 2nd-line & $\begin{array}{l}\text { Post- } \\
\text { autologous } \\
\text { stem cell } \\
\text { transplant }\end{array}$ \\
\hline \multicolumn{9}{|l|}{ PDL-1 inhibitor } \\
\hline Atezolizumab & & 2nd-line & & & 2nd-line & & & \\
\hline Avelumab & & & & & & 2nd-line & & \\
\hline Durvalumab & & $\begin{array}{l}\text { Post } \\
\text { chemotherapy or } \\
\text { radiation for } \\
\text { stage III disease }\end{array}$ & & & 2nd-line & & & \\
\hline \multicolumn{9}{|c|}{ Combination (CTLA-4 + PD-1) } \\
\hline $\begin{array}{l}\text { Ipilimumab + } \\
\text { nivolumab }\end{array}$ & 1st-line & & 1st-line & & & & & \\
\hline
\end{tabular}

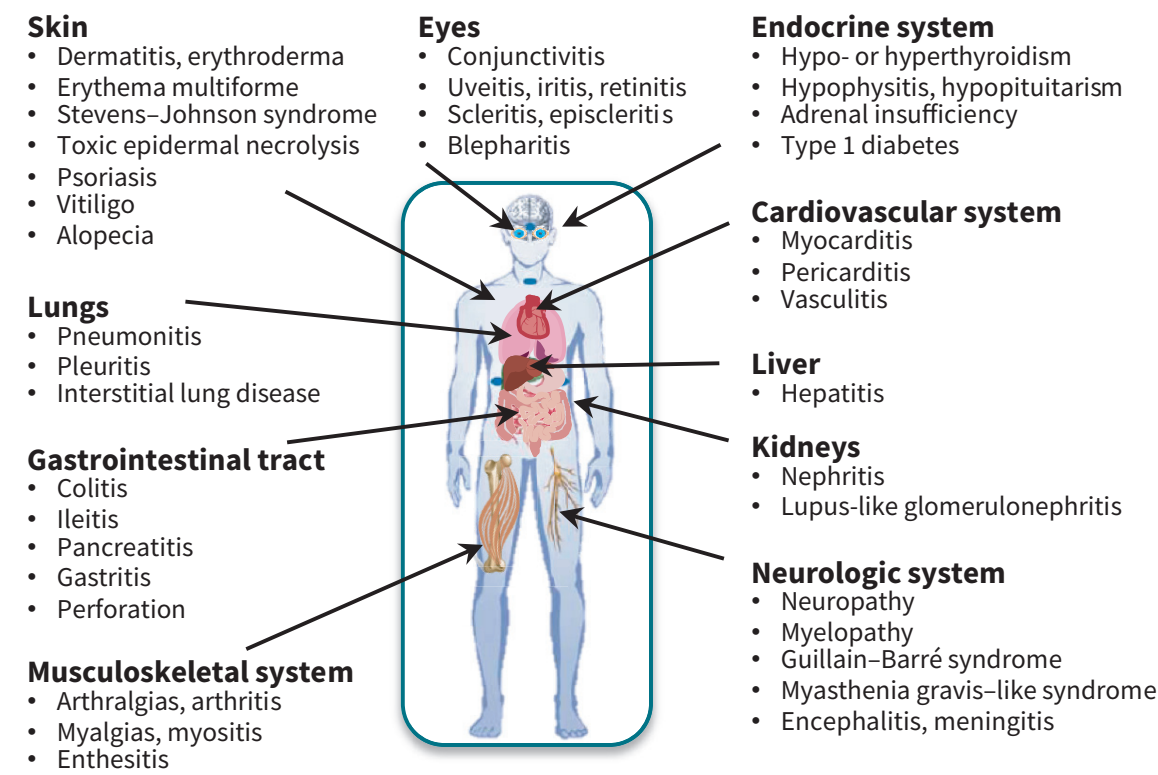

Figure 2: Organs affected by and manifestations of immune-related adverse events. 
Table 2: Rates of the more common immune-related adverse events stratified by immune checkpoint inhibitor strategy

\begin{tabular}{|c|c|c|c|c|c|c|}
\hline \multirow[b]{2}{*}{ Immune-related adverse events } & \multicolumn{2}{|c|}{$\begin{array}{l}\text { Anti-CTLA-4 } \\
\text { (ipilimumab) }\end{array}$} & \multicolumn{2}{|c|}{$\begin{array}{l}\text { Anti-PD-1 } \\
\text { (nivolumab) }\end{array}$} & \multicolumn{2}{|c|}{$\begin{array}{c}\text { Anti-CTLA4 + Anti-PD1 } \\
\text { (ipilimumab + nivolumab) }\end{array}$} \\
\hline & Any grade, $\%$ & Grade $\geq 3, \uparrow \%$ & Any grade, $\%$ & Grade $\geq 3, \uparrow \%$ & Any grade, $\%$ & Grade $\geq 3, \uparrow \%$ \\
\hline Rash & 33 & 2 & 26 & 1 & 40 & 5 \\
\hline Colitis & 12 & 9 & 1 & 1 & 12 & 8 \\
\hline Hepatitis & 4 & 2 & 4 & 1 & 18 & 8 \\
\hline Hypothyroidism & 4 & 0 & 9 & 0 & 15 & 1 \\
\hline $\begin{array}{l}\text { Discontinuation owing to immune- } \\
\text { related adverse events }\end{array}$ & 15 & 13 & 8 & 5 & 36 & 30 \\
\hline
\end{tabular}

\section{Table 3: General grading guidelines from the Common Terminology Criteria for Adverse Events ${ }^{\star 12}$}

\section{CTCAE grade}

1

2

3

4

5

\section{Description}

Mild; asymptomatic or mild symptoms; clinical or diagnostic observations only; intervention not indicated.

Moderate; minimal, local or noninvasive intervention indicated; limiting age appropriate instrumental activity of daily living. Severe or medically significant but not immediately life-threatening; hospitalization indicated; disabling; limiting self care. Life-threatening consequences; urgent intervention indicated.

Death related to adverse event.

Note: $\mathrm{CTCAE}=$ Common Terminology Criteria for Adverse Events.

${ }^{*}$ CTCAE provides general grading guidelines as described within this table. For each specific organ affected, the CTCAE also provides descriptive organ-specific grading. ${ }^{12}$

Oral corticosteroids $(0.5-1 \mathrm{mg} / \mathrm{kg})$ are recommended for grade 2 reactions, while treatment with immune checkpoint inhibitors is held. Once a grade 2 reaction has subsided to grade 1 or resolved and steroids have been tapered and stopped, immune checkpoint inhibitors can be resumed the same dose and schedule.

Grade 3 or higher reactions are managed with high-dose oral or intravenous corticosteroids (prednisone $1 \mathrm{mg} / \mathrm{kg}$ or methylprednisolone $2 \mathrm{mg} / \mathrm{kg}$ ). Intravenous therapy is the preferred route for severe grade $\geq 3$ reaction owing to faster onset of action. Patients should be monitored daily until resolution of symptoms, either in hospital or by outpatient visits. Escalation of immunosuppressive treatment is recommended for grade 3 or higher reactions that fail to improve within 48 to 72 hours of high-dose corticosteroid therapy. Tumour necrosis factor inhibitors such as infliximab are preferred as a second-line immunosuppressant. Other drugs that inhibit T cells, including mycophenolate mofetil, have also been used with success. ${ }^{10}$ In most cases, grade 3 or higher reactions are considered grounds for permanent discontinuation of immune checkpoint inhibitors. Corticosteroids should be tapered slowly, in most cases no faster than over 4 weeks, as flare-ups are common if immunosuppression is tapered too quickly. As with any immunosuppressive treatment, appropriate prophylaxis against opportunistic infections and monitoring patients for other corticosteroid adverse effects are important.

Endocrine immune-related adverse events (i.e., hypothyroidism, adrenal insufficiency, hypogonadism and hypophysitis) are the only adverse events that do not require permanent discontinuation of immune checkpoint inhibitors, regardless of the severity of the reaction, as long as patients achieve a stable clinical status on physiologic hormonal replacement therapy. ${ }^{10}$

One of the most important reasons for complications and morbidity from immune-related adverse events is delay in the prompt administration of corticosteroids. Because immune-related adverse events (e.g., colitis, pneumonitis, encephalitis) are sometimes difficult to distinguish from infectious complications of the same organs (e.g., infectious colitis, pneumonia, meningitis), steroids are often delayed while invasive procedures (e.g., colonoscopy, bronchoscopy, lumbar puncture) are planned and microbiology results are awaited. However, for patients with severe grade 


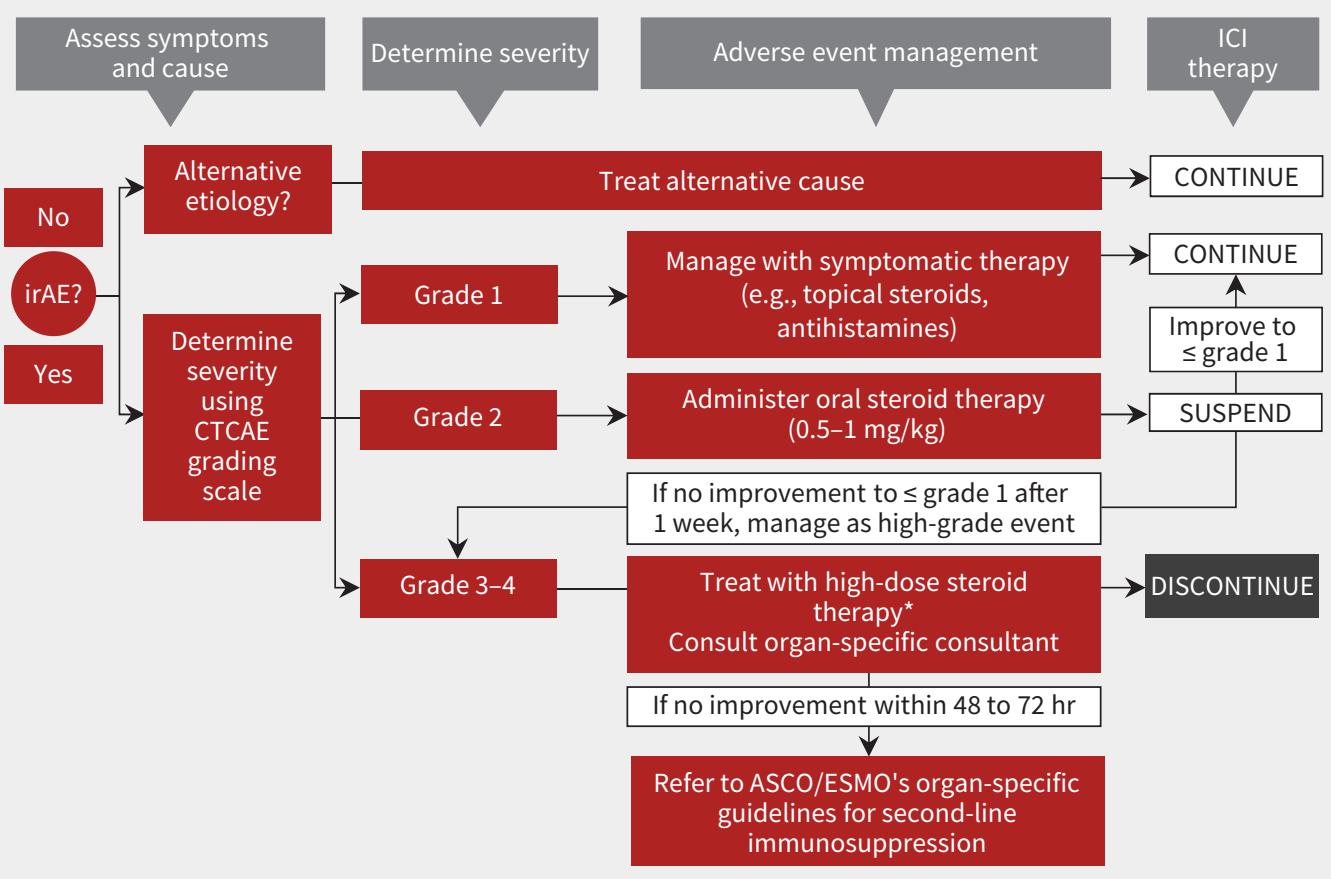

Figure 3: Summarized management strategies for immune-related adverse events (irAE), adapted from the latest American Society of Clinical Oncology (ASCO) guideline..$^{10}$ The only exception to this algorithm is the management of endocrine irAE, where physiologic hormone replacement, rather than high-dose steroid use, is recommended for any grade irAE. *High-dose steroids, defined as $1 \mathrm{mg} / \mathrm{kg}$, or oral prednisone or $2 \mathrm{mg} / \mathrm{kg}$ of intravenous solumedrol. Note: $\mathrm{CTCAE}=$ Common Terminology Criteria for Adverse Events, ESMO = European Society for Medical Oncology, ICI $=\mathrm{immune}$ checkpoint inhibitors.

$\geq 3$ reactions, prompt high-dose intravenous corticosteroid therapy should not be delayed in favour of other investigations. Invasive diagnostic tests and confirmatory biopsies are often challenging to obtain and can lead to complications or delays in treatment. For instance, diagnostic colonoscopies in the setting of colitis from immune checkpoint inhibitors could induce bowel perforation, and should not be routinely performed. ${ }^{13}$

\section{What is the impact of immune-related adverse events on cancer outcomes?}

Early clinical observations suggested that patients with severe immune-related adverse events seemed to derive greater antitumour benefit, sometimes lasting long after the immune checkpoint inhibitors were permanently discontinued. Thus, the occurrence of immune-related adverse events was thought to be tangible proof that the patient's immune system was activated. Whether this immunological activation correlates with improved cancer outcomes remains controversial. For instance, in one large, retrospective study, cancer outcomes were similar in patients with melanoma treated with the cytotoxic T-lymphocyte-associated protein 4 inhibitor ipilimumab whether they experienced immune-related adverse events or not. ${ }^{14}$ However, the development of a specific immune-related adverse event, vitiligo, has been associated with improved cancer outcomes in the same group of patients. ${ }^{15}$ In patients with melanoma who were treated with both ipilimumab and nivolumab, serious toxicities leading to early discontinuation have been associated with good overall survival. ${ }^{16}$ In 2 recent series, the development of immune-related adverse events in patients with lung cancer who were undergoing therapy with immune checkpoint inhibitors was associated with improved survival. ${ }^{17,18}$ In summary, the current consensus is that immunerelated adverse events are not required to obtain a benefit from immune checkpoint inhibitors, but their occurrence is potentially associated with improved cancer-related outcomes in some settings. Further research and large-scale cohort studies are required to investigate this association.

\section{Are immune checkpoint inhibitors suitable for patients with pre-existing autoimmune diseases?}

Patients with autoimmune diseases have been excluded from clinical trials investigating immune checkpoint inhibitors owing to concerns regarding disease flare-ups. In the real-life clinical setting, clinicians have offered immune checkpoint inhibitors to these patients, based on the assumption that the benefits may outweigh the risks. There is some, albeit limited, evidence to support this. These patients seem to derive the same amount of clinical benefit from immune checkpoint inhibitors as the standard populations in which these therapies were studied. ${ }^{19-21}$ As 
far as the autoimmune outcomes are concerned, a systematic review concluded that flare-ups and immune-related adverse events in patients with autoimmune disease on immune checkpoint inhibitors can often be managed without discontinuing therapy, although some events may be severe and fatal. ${ }^{22}$ The optimal immunosuppressive regimen necessary to maintain quiescence of pre-existing autoimmunity without compromising the clinical benefits of immunotherapies has yet to be elucidated. As an example, certain immunomodulatory regimens could be associated with a loss of clinical benefit of immune checkpoint inhibitors. ${ }^{23}$

The management of patients who have cancer and preexisting autoimmune diseases and who are receiving immune checkpoint inhibitors is complex and requires a multidisciplinary approach that incorporates oncologists and organ-specific experts, including rheumatologists, gastroenterologists, endocrinologists and dermatologists.

\section{Can we predict which patients will develop immune-related adverse events?}

There are large gaps in the epidemiology of immune-related adverse events. Demographic risk factors for the development of these events are unknown, including whether female sex, which is a strong risk factor for autoimmunity, is also a risk factor for immune-related adverse events. Although the gut microbiome has been linked to the development of immune checkpoint inhibitor-induced colitis and an intriguing association between response to these inhibitors and gut microbiome has been described, ${ }^{24-26}$ associations between the microbiome outside the gut and immune-related adverse events remain unexplored. The molecular mechanisms underlying immunerelated adverse events, and whether they are similar or not to those underlying "classic" autoimmune diseases, are also largely unknown. Genetic susceptibilities, particularly major histocompatibility complex haplotypes, are mostly absent from current analyses for immune-related adverse events. ${ }^{27}$ Cytotoxic T-lymphocyte-associated protein 4 polymorphisms have been linked to an increased risk of autoimmune diseases and programmed cell death 1 polymorphisms to autoimmune colitis; mice lacking programmed cell death 1 develop lupus-like syndrome. ${ }^{28-31}$ To date, only limited lupus nephritis, rather than full systemic lupus syndrome, has been reported with the use of immune checkpoint inhibitors. ${ }^{32}$ As such, the effect of genetic variants of cytotoxic T-lymphocyte-associated protein 4, programmed cell death 1 , and programmed death-ligand 1 on the risk or severity of immune-related adverse events is unknown. Autoantibodies have been reported in some cases of type 1 diabetes, thyroid disease and arthritis secondary to immune checkpoint inhibitors. ${ }^{33-36}$ Whether these antibodies represent a pre-existing immune diathesis or are the consequence of de novo immunological events remains unknown. Few clinical predictors of immune-related adverse events have been identified, including pre-treatment lymphopenia. ${ }^{37,38}$ Although immune biomarkers predictive of the outcome of treatment with immune checkpoint inhibitors are the object of considerable

\section{Box 2: Unanswered questions}

- Can we predict immune-related adverse events and personalize decision-making regarding treatment with immune checkpoint inhibitors?

- What is the role of the human microbiome in the development of immune-related adverse events?

- Why do some patients have one immune-related adverse event but others have another when the exposure (e.g., anti-cytotoxic T-lymphocyte-associated protein 4) is the same?

- What is the optimal immunosuppressive treatment that will not mitigate the benefits of the treatment?

research, there is a paucity of research on immune biomarkers predicting immune-related adverse events. Unanswered questions are summarized in Box 2.

\section{Conclusion}

Immune checkpoint inhibitors have rapidly established themselves as a new pillar in cancer therapy alongside chemotherapy, radiotherapy and surgery. Some patients previously deemed incurable are now achieving long-term remissions with these novel therapeutics, but at the expense of frequent and sometimes fatal immunerelated adverse events. These adverse events can be challenging to both diagnose and manage. A high clinical suspicion and multidisciplinary approach are often necessary to optimize clinical outcomes. Deciphering the mechanisms driving the immune-related adverse events is a great opportunity for novel research and will likely shed new insights on the interplay between autoimmunity and oncogenesis. As more advances are made in the field and experience with immune checkpoint inhibitors increases, front-line health care workers will be increasingly involved in managing patients who are being treated with these agents.

\section{References}

1. Schadendorf D, Hodi FS, Robert C, et al. Pooled analysis of long-term survival data from Phase II and Phase III Trials of Ipilimumab in Unresectable or Metastatic Melanoma. J Clin Oncol 2015;33:1889-94.

2. Gettinger S, Horn L, Jackman D, et al. Five-year follow-up of nivolumab in previously treated advanced non-small-cell lung cancer: results from the CA209003 study. J Clin Oncol 2018;36:1675-84.

3. Schreiber RD, Old LJ, Smyth MJ. Cancer immunoediting: integrating immunity's roles in cancer suppression and promotion. Science 2011;331:1565-70.

4. Pardoll DM. The blockade of immune checkpoints in cancer immunotherapy. Nat Rev Cancer 2012;12:252-64.

5. Ribas A, Wolchok JD. Cancer immunotherapy using checkpoint blockade. Science 2018;359:1350-5.

6. Larkin J, Chiarion-Sileni V, Gonzalez R, et al. Combined nivolumab and ipilimumab or monotherapy in untreated melanoma. N Engl J Med 2015;373:23-34.

7. Abdel-Wahab N, Shah M, Suarez-Almazor ME. Adverse events associated with immune checkpoint blockade in patients with cancer: a systematic review of case reports. PLoS One 2016;11:e0160221.

8. Sznol M, Ferrucci PF, Hogg D, et al. Pooled analysis safety profile of nivolumab and ipilimumab combination therapy in patients with advanced melanoma. $J$ Clin Oncol 2017;35:3815-22.

9. Desforges P, Esfahani K, Bouganim N. Programmed cell death ligand 1-induced coma from diffuse cerebritis. J Oncol Pract 2018;14:134-5.

10. Brahmer JR, Lacchetti C, Schneider BJ, et al. Management of immune-related adverse events in patients treated with immune checkpoint inhibitor therapy: American Society of Clinical Oncology Clinical Practice Guideline. J Clin Oncol 2018;36:1714-68 
11. Haanen JB, Carbonnel F, Robert C, et al. Management of toxicities from immunotherapy: ESMO Clinical Practice Guidelines for diagnosis, treatment and follow-up. Ann Oncol 2018;29(Suppl 4):iv264-6.

12. Common Terminology Criteria for Adverse Events (CTCAE) version 5.0. Washington (DC): US Department of Health and Human Services; 2017. Available: https://ctep .cancer.gov/protocolDevelopment/electronic_applications/docs/CTCAE_v5_Quick _Reference_5x7.pdf (accessed 2018 Dec. 3).

13. Burdine L, Lai K, Laryea JA. Ipilimumab-induced colonic perforation. J Surg Case Rep 2014;2014:pii: rju010.

14. Horvat TZ, Adel NG, Dang TO, et al. Immune-related adverse events, need for systemic immunosuppression, and effects on survival and time to treatment failure in patients with melanoma treated with ipilimumab at memorial sloan kettering cancer center. J Clin Oncol 2015;33:3193-8.

15. Hua C, Boussemart L, Mateus C, et al. Association of vitiligo with tumor response in patients with metastatic melanoma treated with pembrolizumab. JAMA Dermatol 2016;152:45-51.

16. Shoushtari AN, Friedman CF, Navid-Azarbaijani P, et al. Measuring toxic effects and time to treatment failure for nivolumab plus ipilimumab in melanoma. JAMA Oncol 2018;4:98-101.

17. Haratani K, Hayashi H, Chiba Y, et al. Association of immune-related adverse events with nivolumab efficacy in non-small-cell lung cancer. JAMA Oncol 2018;4:374-8.

18. Sato K, Akamatsu H, Murakami E, et al. Correlation between immune-related adverse events and efficacy in non-small cell lung cancer treated with nivolumab. Lung Cancer 2018;115:71-4.

19. Menzies AM, Johnson DB, Ramanujam S, et al. Anti-PD-1 therapy in patients with advanced melanoma and preexisting autoimmune disorders or major toxicity with ipilimumab. Ann Oncol 2017;28:368-76.

20. Johnson DB, Sullivan RJ, Ott PA, et al. Ipilimumab therapy in patients with advanced melanoma and preexisting autoimmune disorders. JAMA Oncol 2016;2:234-40.

21. Gutzmer R, Koop A, Meier F, et al. Programmed cell death protein-1 (PD-1) inhibitor therapy in patients with advanced melanoma and preexisting autoimmunity or ipilimumab-triggered autoimmunity. Eur J Cancer 2017;75:24-32.

22. Abdel-Wahab N, Shah M, Lopez-Olivo MA, et al. Use of immune checkpoint inhibitors in the treatment of patients with cancer and preexisting autoimmune disease: a systematic review. Ann Intern Med 2018;168:121-30.

23. Esfahani K, Miller WH Jr. Reversal of autoimmune toxicity and loss of tumor response by interleukin-17 blockade. N Engl J Med 2017;376:1989-91.

24. Dubin K, Callahan MK, Ren B, et al. Intestinal microbiome analyses identify melanoma patients at risk for checkpoint-blockade-induced colitis. Nat Commun 2016; 7:10391.
25. Gopalakrishnan V, Spencer CN, Nezi L, et al. Gut microbiome modulates response to anti-PD-1 immunotherapy in melanoma patients. Science 2018;359: 97-103.

26. Routy B, Le Chatelier E, Derosa L, et al. Gut microbiome influences efficacy of PD-1-based immunotherapy against epithelial tumors. Science 2018;359:91-7.

27. Chowell D, Morris LGT, Grigg CM, et al. Patient HLA class I genotype influences cancer response to checkpoint blockade immunotherapy. Science 2018;359: 582-7.

28. Klocke K, Sakaguchi S, Holmdahl R, et al. Induction of autoimmune disease by deletion of CTLA-4 in mice in adulthood. Proc Natl Acad Sci U S A 2016;113:E2383-92.

29. Lühder F, Hoglund P, Allison JP, et al. Cytotoxic T lymphocyte-associated antigen 4 (CTLA-4) regulates the unfolding of autoimmune diabetes. J Exp Med 1998;187: 427-32.

30. Nishimura H, Nose M, Hiai H, et al. Development of lupus-like autoimmune diseases by disruption of the PD-1 gene encoding an ITIM motif-carrying immunoreceptor. Immunity 1999;11:141-51.

31. Liu JZ, van Sommeren S, Huang H, et al. Association analyses identify 38 susceptibility loci for inflammatory bowel disease and highlight shared genetic risk across populations. Nat Genet 2015;47:979-86.

32. Fadel F, El Karoui K, Knebelmann B. Anti-CTLA4 antibody-induced lupus nephritis. N Engl J Med 2009;361:211-2.

33. Godwin JL, Jaggi S, Sirisena I, et al. Nivolumab-induced autoimmune diabetes mellitus presenting as diabetic ketoacidosis in a patient with metastatic lung cancer. J Immunother Cancer 2017;5:40.

34. Osorio JC, Ni A, Chaft JE, et al. Antibody-mediated thyroid dysfunction during T-cell checkpoint blockade in patients with non-small-cell lung cancer. Ann Oncol 2017;28:583-9.

35. Belkhir R, Burel SL, Dunogeant L, et al. Rheumatoid arthritis and polymyalgia rheumatica occurring after immune checkpoint inhibitor treatment. Ann Rheum Dis 2017;76:1747-50.

36. Kostine M, Rouxel L, Barnetche $\mathrm{T}$, et al. Rheumatic disorders associated with immune checkpoint inhibitors in patients with cancer-clinical aspects and relationship with tumour response: a single-centre prospective cohort study. Ann Rheum Dis 2018;77:393-8.

37. Diehl A, Yarchoan M, Hopkins A, et al. Relationships between lymphocyte counts and treatment-related toxicities and clinical responses in patients with solid tumors treated with PD-1 checkpoint inhibitors. Oncotarget 2017;8: 114268-80.

38. Suh KJ, Kim SH, Kim YJ, et al. Post-treatment neutrophil-to-lymphocyte ratio at week 6 is prognostic in patients with advanced non-small cell lung cancers treated with anti-PD-1 antibody. Cancer Immunol Immunother 2018;67:459-70.
Competing interests: Marie Hudson reports receiving a grant from BristolMyers Squibb, outside the submitted work. Khashayar Esfahani reports receiving speaker's fees from Bristol-Myers Squibb, outside the submitted work. Wilson Miller reports receiving grants from Bristol-Myers Squibb, Merck, Roche, Novartis, AstraZeneca, Amgen, Bayer, Medlmmune and GlaxoSmithKline, outside the submitted work, as well as personal fees from Bristol-Myers Squibb, Merck, Roche, Novartis, Amgen and GlaxoSmithKline. No other competing interests were declared.

This article was solicited and has been peer reviewed.

Affiliations: Department of Medicine (Esfahani, Meti, Miller, Hudson), McGill University; Departments of Medicine and Oncology (Esfahani,
Miller), McGill University and Jewish General Hospital, Rossy Cancer Network; Division of Rheumatology and Lady Davis Institute (Hudson), Jewish General Hospital, Montreal, Que.

Contributors: Khashayar Esfahani and Nicholas Meti were joint primary authors. All of the authors contributed to the conception and design of the work, and the acquisition, analysis and interpretation of data. All of the authors drafted the manuscript, revised it critically for important intellectual content, gave final approval of the version to be published and agreed to be accountable for all aspects of the work.

Correspondence to: Marie Hudson, marie.hudson@mcgill.ca; or Khashayar Esfahani, khashayar.esfahani@mail.mcgill.ca 